\title{
Association of Tear Matrix Metalloproteinase 9 Immunoassay With Signs and Symptoms of Dry Eye Disease: A Cross-Sectional Study Using Qualitative, Semiquantitative, and Quantitative Strategies
}

\section{You Hyun Lee}

Keimyung University Dongsan Medical Center

\section{Seung-Pil Bang}

University of Rochester

Kyu-Young Shim

Keimyung University Dongsan Medical Center

Myung-Jin Son

Keimyung University Dongsan Medical Center

Harim Kim

Keimyung University Dongsan Medical Center Jong Hwa Jun ( $\sim$ junjonghwa@gmail.com ) Keimyung University Dongsan Medical Center

\section{Research Article}

Keywords: Matrix Metalloproteinase, Qualitative, Semiquantitative, Quantitative Strategies

Posted Date: December 16th, 2020

DOI: https://doi.org/10.21203/rs.3.rs-121664/v1

License: (c) (i) This work is licensed under a Creative Commons Attribution 4.0 International License.

Read Full License 


\section{Abstract}

This study aimed to analyze the association of tear matrix metalloproteinase 9 (MMP-9) immunoassay with the severity of dry eye (DE) signs and symptoms through qualitative, semiquantitative, and quantitative evaluations of immunoassay band. This cross-sectional study enrolled 320 eyes of 320 patients. The clinical signs of DE were assessed using the Ocular Surface Disorder Index (OSDI) score, visual analogue scale (VAS), tear breakup time (tBUT), tear volume evaluation by tear meniscometry, and staining scores of the cornea and conjunctiva by the Oxford grading scheme. Positive MMP-9 immunoassay results were significantly related to shorter tBUT, tBUT $\leq 3$ seconds, higher corneal staining score, corneal staining score $\geq 2$, and conjunctival staining score $\geq 2$. The semiquantitative results of the MMP-9 immunoassay were positively correlated with higher corneal staining score and negatively correlated with tBUT. However, in the quantitative analysis, none of the DE signs or symptoms were correlated to the band density of the MMP-9 immunoassay. In conclusion, The qualitatively and semiquantitatively evaluated MMP-9 immunoassay results are related to the clinical severity of DE. However, the quantitatively evaluated MMP-9 immunoassay results did not represent the clinical severity of DE disease.

\section{Introduction}

Dry eye (DE) is a multifactorial disease of the tears and ocular surface that results in visual disturbance and tear film instability, and it negatively impacts daily living, emotional well-being, and the ability to work $[1,2]$. Until now, clinical assessment by Schirmer test and tear breakup time (tBUT) have been used to diagnose and assess DE severity 3,4 [3]. However, as inflammation is one of the core mechanisms in the development of DE, new diagnostic tools have emerged for detecting ocular surface inflammation [5], such as the InflammaDry (Quidel Corporation, San Diego, CA, USA) [6-8]. This assay can detect the presence of matrix metalloproteinase 9 (MMP-9) with a concentration $>40 \mathrm{ng} / \mathrm{mL}$ in tears.

Various studies were performed to validate this new point-of-care MMP-9 immunoassay. Such studies mostly focused on the comparison of qualitative test results with the clinical symptoms of DE. Messmer et al. [9] reported that decreased tBUT, severe meibomian gland dysfunction (MGD), ocular surface staining, and low Schirmer test results were significantly correlated with positive MMP-9 results. Chotikavanich et al. [10] mentioned that MMP-9 positivity is significantly correlated with the symptom severity scores, topographic surface regularity index, conjunctival and corneal fluorescein staining scores, and tBUT.

MMP-9 is produced by corneal epithelium, and proinflammatory cytokines, such as tumor necrosis factor a, interleukin 1 , and tumor growth factor $\beta 1$, aggravate ocular surface inflammation in DE $[5,11]$. Based on this, it can be assumed that worse ocular surface inflammation induces stronger antigen-antibody reaction, which results in stronger band density in MMP-9 immunoassay. However, studies on the relationship between MMP-9 and the clinical severity of DE are lacking 


\section{Methods}

\section{Aim, Study Design, and Setting}

This study aimed to investigate the MMP-9 immunoassay results using qualitative, semiquantitative, and quantitative evaluations of immunoassay band and to evaluate the correlation between each test result and the clinical signs and symptoms of DE. This cross-sectional study enrolled 320 patients who visited our ophthalmic department from April 1, 2017, through October 1, 2018. The study adhered to the tenets of the Declaration of Helsinki and was approved by the institutional review board of Keimyung University Dongsan Hospital (IRB no. 2017-06-008). The patients signed informed consent for the use of their data. The study investigator collected clinical data and MMP-9 test results from the right eye of each enrolled patient.

\section{Clinical Assessment of DE and Data Collected}

\section{Enrolled criteria}

Patients with chief complaints of DE symptoms such as stinging, burning, and/or scratchy sensation in eyes and met at least one of the following four criteria were recruited: Ocular Surface Disease Index (OSDI) score $>20$, tBUT $<5$ seconds, tear meniscometry test results without anesthesia $<5 \mathrm{~mm} / 5$ seconds, and corneal fluorescein staining results $\geq 1$. Clinical evaluations were performed in the following sequence: OSDI questionnaire/visual analogue scale (VAS) score, tear meniscometry, tear MMP-9 immunoassay, tBUT, corneal and conjunctival staining scores, and meibomian gland evaluation after topical proparacaine (Paracaine; Hanmi Pharm, Seoul, South Korea) instillation. Patients with active ocular infection or inflammation, who were pregnant, or lacrimal drainage disorders such as lacrimal punctal stenosis, deformed lacrimal punctum, canalicular anomalies, and nasolacrimal duct obstruction; those receiving topical or systemic corticosteroid treatment or immunomodulatory therapy within 1 month; those who had fluorescein allergy, cornstarch or dacron allergy, undergone ocular surgery within 6 months, or had ocular trauma in the previous 3 months; and those who wore contact lenses within 72 hours were excluded.

\section{Subjective symptoms}

Patients underwent full ophthalmologic examination. Subjective symptoms were measured using the OSDI questionnaire [12]. OSDI scores range from 0 to 100 , where 0 indicates no disability and 100 indicates complete disability. The degree of ocular pain was documented using the VAS, where 0 indicates no pain and 10 indicates worst possible pain [13].

\section{Tear Strip Meniscometry for Tear Volume Evaluation}

The tear volume was measured by tear meniscometry (SMTube®; Echo Electricity Co., Ltd., Fukushima, Japan). After blinking voluntarily 2 to 3 times, the tip of the tear meniscometry tube was applied to the 
lateral third area of the lower lid tear lake for 5 seconds. Tear volume was recorded by the length (in millimeters) of the stained tear column.

\section{Tear MMP-9 Point-of-care Test}

The tear MMP-9 immunoassay (InflammaDry) test was performed according to the manufacturer's instruction by a single examiner ( $\mathrm{JHJ})$. To collect a tear sample, the sample collector was dabbed three times in three different locations of the inferior conjunctival palpebrae (temporal, middle, nasal; from nasal to temporal direction) and was placed against the temporal inferior palpebral conjunctiva for an additional 5 seconds. After that, the sample collector was snapped to a test cassette. After 5 seconds, the absorbent tip was immersed into a buffer solution. To evaluate the result band density of the test line under the same conditions, a photograph of the result window was taken with a slit-lamp biomicroscope mounted with a single-lens reflex camera (Canon EOS 700D, setting: ISO 400, shutter speed 1/200 sec; Canon USA, Melville, NY, USA) 20 minutes after the test initiation. Test results were interpreted using qualitative, semiquantitative, and quantitative. Qualitative analysis was performed using a two-point scale: $0=$ negative, $1=$ positive). Results were considered assay positive when there were other bands aside from than the faint band. Semiquantitative analysis based on the colour intensity of the test (red) line was performed by a single clinician ( $\mathrm{JHJ}$ ) using a four-point scale: $0=$ negative; $1=$ weak positive; 2=moderate positive; 3 =strong positive (Figure 1). Quantitative analysis of red line band density was measured with ImageJ version 1.44p (National Institutes of Health, Bethesda, MD, USA) by three different experimenters (YHL, M-JS, and HK) [14]. First, the image of the test result was mounted to the ImageJ software and converted to 8-bit colour. Second, a square that included both the reagent and control bands was drawn. Third, the first lane was selected, followed by the select plot lanes in the analyze tool. Fourth, two peak points were checked, and two lines that constituted the most ideal parabola were drawn. Lastly, the wand tool was select, and the area that represents the reagent (Figure 2a) and control band (Figure $2 b)$ densities was clicked. The quantitative results were calculated by the ratio of the reagent band density to the control band density from ImageJ.

\section{tBUT}

The tBUT was assessed by a wetted fluorescein strip (Haag-Streit AG, Koeniz, Switzerland) touched into the lower inferotemporal bulbar conjunctiva. Patients were instructed to blink, and the interval time between the last blink and the first appearance of dark spots in the tear film was recorded using a stopwatch under blue-light illumination with a biomicroscope and x10 magnification.

\section{Corneal and Conjunctival Stain Scores}

Corneal and conjunctival staining was conducted using fluorescein instillation into the tear film, and the score was measured using the Oxford grading scheme. The degree of staining was based on the number of dots on series of panels (A-E); the staining score ranges from 0 to 5 for each panel, for a total possible score ranging from 0 to 15 for the exposed interpalpebral conjunctiva and cornea [15]. The conjunctival 
stain score was measured for the nasal and temporal sides of the right eye, and the total score was used for the correlation analysis.

\section{Meibomian Gland Evaluation}

MGD was assessed according to its secretion turbidity and expressibility. Turbidity was graded from 0 to 3: clear $=0$; cloudy $=1$; granular=2; inspissated $=3$. Expressibility was assessed after application of topical anesthesia and graded from 0 to 3: $0=$ clear meibum with easily expressed, $1=$ cloudy meibum expressed with mild pressure, $2=$ thick cloudy meibum expressed with more than moderate pressure; $3=$ meibum not expressed, even with hard pressure $[16,17]$.

\section{Statistical methods}

Data were calculated as mean \pm standard deviation (SD), unless otherwise specified. Statistics were analyzed using SPSS version 12.0 (IBM, Chicago, IL, USA). The between-group differences in age, OSDI score, VAS score, tear meniscometry, tBUT, corneal staining, conjunctival staining, turbidity, and expressibility of the meibomian gland were compared using independent t-test. The relationship of various systemic diseases related to the DE and MMP-9 positivity was analyzed using chi-square test. The Spearman correlation test was performed between semiquantitative MMP-9 results and age, OSDI score, VAS score, tear meniscometry results, tBUT, corneal staining score, conjunctival staining score, and MGD score. The Pearson correlation test was performed between quantitative results of MMP-9 and other age, OSDI score, VAS score, tear meniscometry results, tBUT, corneal staining score, conjunctival staining score, and MGD score. Two-sided $p$ values $<0.05$ were considered statistically significant

\section{Results}

\section{Qualitative Results of the MMP-9 Immunoassay}

\section{Demographics of the study populations}

A total of 320 patients (320 eyes) were included in the study. The mean age was $58 \pm 13$ years (range, $14-$ 90 years) and 97 (30\%) were men. One hundred eighty-two patients had confirmed DE. The demographic data of patients according to MMP-9 positivity are shown in Table 1. There are no significant differences between the groups, and the presence of systemic autoimmune disease and Sjögren disease was not significantly different either ( $p>0.05)$.

Comparisons between the qualitative results of the MMP-9 immunoassay and symptoms of dry eye

There were no statistically significant differences in OSDI and VAS between the MMP-9-positive and MMP-9-negative groups (Table 2).

Tear volume measurements by tear meniscometry 
There was no statistically significant difference in mean tear volume, as measured by tear meniscometry, between the MMP-9-positive and MMP-9-negative groups (Table 2).

\section{Tear breakup time}

The mean tBUT of the MMP-9-positive group was shorter than that of the MMP-9-negative group, and it was statistically significant. Furthermore, when stratified by tBUT <3 seconds, the MMP-9-positive group showed significantly shorter tBUT than the MMP-9-negative group (Table 2).

\section{Corneal and conjunctival stain score}

The mean corneal stain score of the MMP-9-positive group was higher, and it was statistically significant. When it was stratified by a corneal stain score $>2$, significantly more patients were observed in the MMP-9-positive group than that in the MMP-9-negative group (Table 2). In addition, the mean conjunctival stain scores of the MMP-9-positive and MMP-9-negative groups were not significantly different. When stratified by a conjunctival staining score $>2$, significantly more patients were observed in the MMP-9-positive group than that in the MMP-9-negative group (Table 2).

$M G D$

The mean meibomian gland turbidity in the MMP-9-negative group was slightly higher than that of the MMP-9-positive group, but the difference was not statistically significant. The grades of meibomian gland expression were not significantly different between the two groups. A higher percentage of MGD grade $>3$ was noted in the MMP-9 positive group, but it was not statistically significant (Table 2).

\section{Semiquantitative and Quantitative Results of the MMP-9 Immunoassay}

\section{Demographics of the study populations}

The demographics of the allocated patients are shown in Table 3. No difference in age, sex, systemic autoimmune disease, Sjögren syndrome, and systemic diseases (diabetes, hypertension, thyroid disease, cardiac disease, sleep disorder, Parkinson disease, other neurologic disease, and pulmonary disease) was observed in the four groups ( $p>0.05)$.

Comparisons between the semiquantitative results of the MMP-9 immunoassay and DE severity

No significant difference in age, OSDI, VAS, Schirmer score, tBUT, tBUT $\leq 3$ seconds, corneal staining score, conjunctival staining score, conjunctival staining score $\geq 2$, meibomian gland turbidity, expression, and dysfunction was observed in the four groups ( $p>0.05)$. However, significant difference was observed in corneal staining score $\geq 2$ (Table 4 ). The subgroup analysis showed significantly higher percentages of corneal staining score $\geq 2$ in grade 1 and 2 groups than in grade 0 group (grade 0 vs. grade 1: $p=0.02$; grade 0 vs. grade 3: $p=0.011$ ).

Clinically significant associations with semiquantitative MMP-9 immunoassay results 
The tBUT showed significant negative correlation with the semiquantitative MMP-9 immunoassay results (Table 5). In contrast, the corneal staining score showed significant positive correlation with the semiquantitative MMP-9 immunoassay results (Table 5). Other parameters, such as age, OSDI score, VAS score, Schirmer score, MGD, and conjunctival staining score, showed nonsignificant correlation (Table 5 , $p>0.05)$.

\section{Clinically significant associations with quantitative MMP-9 immunoassay results}

The age, OSDI, VAS, Schirmer score, tBUT, corneal staining score, conjunctival staining score, and MGD showed clinically nonsignificant correlation with the quantitative MMP-9 immunoassay results (Table 6, $p>0.05)$.

\section{Discussion}

DE is a chronic condition affecting $5 \%$ to $30 \%$ of the population aged 50 years or older [1]. Traditionally, symptoms of DE are evaluated using DE questionnaires, and DE signs are assessed by tBUT, corneal staining score, conjunctival staining score, tear film assessment, and the Schirmer test $[1,18]$. However, the pathogenesis of DE is still not fully understood. During the past 20 years, clinicians have paid more attention to inflammation and recognized its key role in the development and amplification of signs and symptoms of DE ${ }^{19}$ [19]. MMP-9 is a nonspecific biomarker of inflammation, and elevated tear MMP-9 levels were found in DE $[7,10]$. Aragona et al. [20] demonstrated that MMP-9 levels measured by polymerase chain reaction correlated well with corneal or conjunctival stain scores and tBUT. Quantitative analysis of MMP-9 using enzyme-linked immunosorbent assay had been introduced; however, this method is time-consuming and expensive, rendering it difficult to use routinely in clinics [21]. To overcome these drawbacks, the MMP-9 immunoassay (InflammaDry) was developed. This immunoassay has advantages of low cost, rapid results, and ease of device preparation [22].

Although InflammaDry is a point-of-care immunoassay developed to discriminate between DE and non$\mathrm{DE}$, clinicians inevitably became curious about the clinical meaning of weak positive and strong positive results. In addition, the basic principle of InflammaDry is that the band is expressed through a colourimetric reaction based on antigen-antibody binding; thus, there is a possibility that the distinctness of the band may vary in proportion to the concentration of MMP-9, that is, the degree of clinical ocular surface inflammation.

In the present study, we evaluated the result of tear MMP-9 immunoassay test using qualitative, semiquantitative, and quantitative analyses of immunoassay band. In the qualitative analysis, shorter tBUT, tBUT $\leq 3$ seconds, higher corneal staining score, corneal staining score $\geq 2$, and conjunctival staining score $\geq 2$ were related to a positive MMP-9 immunoassay result. This implies that positive MMP9 immunoassay results are correlated to the clinical severity of DE.

In addition, we found that shorter tBUT and higher corneal stain score, which already showed significant differences in the qualitative analysis, were correlated to the semiquantitative MMP-9 immunoassay 
results. Shimazaki-Den et al. [23] showed the close relation of mucin components with tBUT. As mucin plays an important role in maintaining corneal health and is secreted by the goblet cells, and diminished goblet cell density is observed in chronic inflammation such as allergy, the decreased mucin secretion caused by chronic inflammation resulted in stronger band density ${ }^{24}$ [24]. This finding is also consistent with that of Park et al. [25], to some extent, who demonstrated good correlation between semiquantitative MMP-9 grading and DE signs and symptoms. In our results, however, we could not identify any correlation between clinical symptoms and MMP-9 positivity. This is because the symptoms of DE is nonspecific, as similar symptoms can present in various ocular surface diseases, and Pflugfelder et al. [26] reported that only $57 \%$ of symptomatic patients showed clinical signs of DE.

The quantitative analysis using ImageJ showed no correlation between the immunoassay band density and the clinical signs and symptoms of DE. This implies that quantitative analysis of the immunoassay is insufficient for assessing the clinical severity of DE. The reason for the discrepancies between the semiquantitative and quantitative results might be that the semiquantitative results are only based on the interpretation of reagent band density, whereas the quantitative results are based on both the reagent and control band densities. Same reagent band density can be read differently in the semiquantitative and quantitative analyses of MMP-9 immunoassay. Furthermore, our previous study pointed out that the InflammaDry test band density is influenced by tear volume [8,27]. The lack of assessment of individual tear volume might have resulted in missing the correlation between the quantitative test results and the clinical signs and symptoms of DE. Thus, further studies assessing the correlation between the clinical signs and symptoms of DE and the MMP-9 immunoassay should consider the tear volume.

The following limitation must be considered. We could not evaluate the tear MMP-9 levels in this study. Measuring the real concentration of MMP-9 in tears could provide more accurate information for semiquantitative or quantitative analysis. However, we suggest that the quantitative analysis using the ratio of the reagent band density to the control band density can indirectly represent the tear MMP-9 levels.

In conclusion, The qualitatively and semiquantitatively evaluated MMP-9 immunoassay results are related to the clinical severity of DE. However, the quantitatively evaluated MMP-9 immunoassay results did not represent the clinical severity of DE disease.

\section{Declarations}

\section{Data availability}

The datasets generated and/or analysed during the present study are available from the corresponding author on reasonable request.

\section{Acknowledgements}

Not applicable 


\section{Author Contributions}

Y.H.L. conception of work, data analysis and interpretation, and manuscript writing. S.P.B. data acquisition and analysis. K.Y.S. data acquisition and analysis. M.J.S. data acquisition and analysis. H.R.K. data acquisition and analysis. J.H.J. conception of work, manuscript writing, and substantial revision. All authors reviewed and approved the final manuscript.

\section{Corresponding author}

Correspondence to Jong Hwa Jun.

\section{Competing Interests}

The authors declare no competing interests.

\section{References}

1 The definition and classification of dry eye disease: report of the Definition and Classification Subcommittee of the International Dry Eye WorkShop (2007). The ocular surface 5, 75-92 (2007).

2 Pouyeh, B. et al. Impact of ocular surface symptoms on quality of life in a United States veterans affairs population. American journal of ophthalmology 153, 1061-1066.e1063, doi:10.1016/j.ajo.2011.11.030 (2012).

3 Lucca, J. A., Nunez, J. N. \& Farris, R. L. A comparison of diagnostic tests for keratoconjunctivitis sicca: lactoplate, Schirmer, and tear osmolarity. The CLAO journal : official publication of the Contact Lens Association of Ophthalmologists, Inc 16, 109-112 (1990).

4 Korb, D. R. Survey of preferred tests for diagnosis of the tear film and dry eye. Cornea 19, 483-486 (2000).

5 Stern, M. E., Schaumburg, C. S. \& Pflugfelder, S. C. Dry eye as a mucosal autoimmune disease. International reviews of immunology 32, 19-41, doi:10.3109/08830185.2012.748052 (2013).

6 Lanza, N. L. et al. Dry Eye Profiles in Patients with a Positive Elevated Surface Matrix Metalloproteinase 9 Point-of-Care Test Versus Negative Patients. The ocular surface 14, 216-223, doi:10.1016/j.jtos.2015.12.007 (2016).

7 Lanza, N. L., Valenzuela, F., Perez, V. L. \& Galor, A. The Matrix Metalloproteinase 9 Point-of-Care Test in Dry Eye. The ocular surface 14, 189-195, doi:10.1016/j.jtos.2015.10.004 (2016).

8 Jun, J. H., Lee, Y. H., Son, M. J. \& Kim, H. Importance of tear volume for positivity of tear matrix metalloproteinase-9 immunoassay. PLoS One 15, e0235408-e0235408, doi:10.1371/journal.pone.0235408 (2020). 
9 Messmer, E. M., von Lindenfels, V., Garbe, A. \& Kampik, A. Matrix Metalloproteinase 9 Testing in Dry Eye Disease Using a Commercially Available Point-of-Care Immunoassay. Ophthalmology 123, 2300-2308, doi:10.1016/j.ophtha.2016.07.028 (2016).

10 Chotikavanich, S. et al. Production and activity of matrix metalloproteinase-9 on the ocular surface increase in dysfunctional tear syndrome. Investigative ophthalmology \& visual science 50, 3203-3209, doi:10.1167/iovs.08-2476 (2009).

11 Acera, A., Rocha, G., Vecino, E., Lema, I. \& Duran, J. A. Inflammatory markers in the tears of patients with ocular surface disease. Ophthalmic research 40, 315-321, doi:10.1159/000150445 (2008).

12 Schiffman, R. M., Christianson, M. D., Jacobsen, G., Hirsch, J. D. \& Reis, B. L. Reliability and validity of the Ocular Surface Disease Index. Archives of ophthalmology (Chicago, III. : 1960) 118, 615-621 (2000).

13 McCormack, H. M., Horne, D. J. \& Sheather, S. Clinical applications of visual analogue scales: a critical review. Psychological medicine 18, 1007-1019 (1988).

14 Hartig, S. M. Basic image analysis and manipulation in ImageJ. Current protocols in molecular biology Chapter 14, Unit14.15, doi:10.1002/0471142727.mb1415s102 (2013).

15 Bron, A. J., Evans, V. E. \& Smith, J. A. Grading of corneal and conjunctival staining in the context of other dry eye tests. Cornea 22, 640-650 (2003).

16 Arita, R. et al. Proposed diagnostic criteria for obstructive meibomian gland dysfunction. Ophthalmology 116, 2058-2063.e2051, doi:10.1016/j.ophtha.2009.04.037 (2009).

17 Bron, A. J., Benjamin, L. \& Snibson, G. R. Meibomian gland disease. Classification and grading of lid changes. Eye (London, England) 5 ( Pt 4), 395-411, doi:10.1038/eye.1991.65 (1991).

18 Serin, D., Karsloğlu, S., Kyan, A. \& Alagöz, G. A simple approach to the repeatability of the Schirmer test without anesthesia: eyes open or closed? Cornea 26, 903-906, doi:10.1097/IC0.0b013e3180950083 (2007).

19 Stevenson, W., Chauhan, S. K. \& Dana, R. Dry eye disease: an immune-mediated ocular surface disorder. Archives of ophthalmology (Chicago, III. : 1960) 130, 90-100, doi:10.1001/archophthalmol.2011.364 (2012).

20 Aragona, P. et al. Matrix metalloproteinase 9 and transglutaminase 2 expression at the ocular surface in patients with different forms of dry eye disease. Ophthalmology 122, 62-71, doi:10.1016/j.ophtha.2014.07.048 (2015).

21 Hetherington, S. V., Spitznagel, J. K. \& Quie, P. G. An enzyme-linked immunoassay (ELISA) for measurement of lactoferrin. Journal of immunological methods $65,183-190$, doi:10.1016/00221759(83)90314-9 (1983). 
22 Koczula, K. M. \& Gallotta, A. Lateral flow assays. Essays in biochemistry 60, 111-120, doi:10.1042/ebc20150012 (2016).

23 Shimazaki-Den, S., Dogru, M., Higa, K. \& Shimazaki, J. Symptoms, visual function, and mucin expression of eyes with tear film instability. Cornea 32, 1211-1218, doi:10.1097/ICO.0b013e318295a2a5 (2013).

24 Toda, I., Shimazaki, J. \& Tsubota, K. Dry eye with only decreased tear break-up time is sometimes associated with allergic conjunctivitis. Ophthalmology 102, 302-309, doi:10.1016/s0161-6420(95)31024$x(1995)$.

25 Park, J. Y., Kim, B. G., Kim, J. S. \& Hwang, J. H. Matrix Metalloproteinase 9 Point-of-Care Immunoassay Result Predicts Response to Topical Cyclosporine Treatment in Dry Eye Disease. Translational vision science \& technology 7, 31, doi:10.1167/tvst.7.5.31 (2018).

26 Pflugfelder, S. C. et al. Evaluation of subjective assessments and objective diagnostic tests for diagnosing tear-film disorders known to cause ocular irritation. Cornea 17, 38-56, doi:10.1097/00003226199801000-00007 (1998).

27 Bang, S. P., Son, M. J., Kim, H., Lee, Y. H. \& Jun, J. H. In vitro validation of the tear matrix metalloproteinase 9 in-situ immunoassay. Scientific Reports 10, 15126, doi:10.1038/s41598-020-71977w (2020).

\section{Tables}

Table 1. Demographics in MMP-9 positive versus negative patients. 


\begin{tabular}{|c|c|c|c|c|}
\hline Parameters & \multicolumn{2}{|c|}{$\begin{array}{l}\text { MMP-9 positive } \\
(\mathrm{n}=205)\end{array}$} & $\begin{array}{l}\text { MMP-9 negative } \\
(n=115)\end{array}$ & $P$ value \\
\hline Age, mean (SD) & $59(14)$ & & $58(13)$ & 0.484 \\
\hline Gender, n (\%) male & $65(31.7 \%)$ & & $32(27.8 \%)$ & 0.527 \\
\hline $\begin{array}{l}\text { Systemic autoimmune disease } \\
\text { (except Sjögren syndrome), n (\% }\end{array}$ & $20(9.8 \%)$ & & $14(12.2 \%)$ & 0.571 \\
\hline Sjögren syndrome, n (\%) & $26(12.7 \%)$ & & $9(7.8 \%)$ & 0.197 \\
\hline Diabetes, n (\%) & $19(9.3 \%)$ & $5(4.3 \%)$ & 0.125 & \\
\hline Hypertension, n (\%) & $53(25.9 \%)$ & $25(21.7 \%$ & 0.498 & \\
\hline Thyroid disease, n (\%) & $27(13.2 \%)$ & & $15(13.2 \%)$ & 1.000 \\
\hline Cardiac disease, n (\%) & $18(8.8 \%)$ & & $12(10.4 \%)$ & 0.690 \\
\hline Sleep disorder, n (\%) & $16(7.8 \%)$ & & $16(13.9 \%)$ & 0.085 \\
\hline Parkinson disease, n (\%) & $21(10.2 \%)$ & & $8(7.0 \%)$ & 0.418 \\
\hline Other neurologic disease, $\mathrm{n}(\%)$ & $27(13.2 \%)$ & & $14(12.2 \%)$ & 0.863 \\
\hline Pulmonary disease, $n(\%)$ & $10(4.9 \%)$ & & $6(5.2 \%)$ & 1.000 \\
\hline
\end{tabular}

MMP-9, matrix metalloproteinase-9.

Table 2. Comparison of clinical signs and symptoms in MMP-9 positive versus negative patients. 


\begin{tabular}{|llll|}
\hline Parameters & $\begin{array}{l}\text { MMP-9 positive } \\
(\mathbf{n}=\mathbf{2 0 5})\end{array}$ & $\begin{array}{l}\text { MMP-9 negative } \\
(\mathbf{n}=115)\end{array}$ & $P$ value \\
\hline OSDI score, mean (SD) & $35.2(23.7)$ & $33.8(24)$ & 0.628 \\
\hline VAS scale, mean (SD) & $3.1(2.7)$ & $2.6(2.8)$ & 0.106 \\
\hline Schirmer score, mm, mean (SD) & $4.7(2.4)$ & $4.7(2.4)$ & 0.919 \\
\hline tBUT, sec, mean (SD) & $3.9(2.6)$ & $4.8(3.6)$ & $0.020^{\star}$ \\
\hline tBUT, $\leq 3, \mathrm{n}$ (\%) & $123(60.0 \%)$ & $56(48.7 \%)$ & $0.047^{\star}$ \\
\hline Corneal staining, score, mean (SD) & $1.2(1.1)$ & $0.9(1.0)$ & $0.012^{\star}$ \\
\hline Corneal staining, $\geq 2, \mathrm{n}$ (\%) & $94(45.9 \%)$ & $37(32.2 \%)$ & $0.018^{\star}$ \\
\hline Conjunctival staining, score, mean (SD) & $1.6(2.0)$ & $1.2(1.8)$ & 0.058 \\
\hline Conjunctival staining, $\geq 2, \mathrm{n}(\%)$ & $94(45.9 \%)$ & $35(30.7 \%)$ & $0.008^{\star}$ \\
\hline Meibomian gland turbidity, grade, mean (SD) & $1.1(0.7)$ & $1.2(0.6)$ & 0.912 \\
\hline Meibomian gland expression, grade, mean (SD) & $1.3(1.1)$ & $1.2(1.0)$ & 0.362 \\
\hline Meibomian gland dysfunction, $\geq 3, \mathrm{n}(\%)$ & $108(52.7 \%)$ & $53(46.1 \%)$ & 0.411 \\
\hline
\end{tabular}

MMP-9, matrix metalloproteinase-9; OSDI, ocular surface disease index; VAS, visual analogue scale; tBUT, tear break up time

*Statistically significant by independent two-samples t test or Pearson's chi square test.

Table 3. Demographics of semiquantitative results of MMP-9 point-of-care test. 


\begin{tabular}{|c|c|c|c|c|c|}
\hline Parameters & $\begin{array}{l}\text { Grade } \\
0 \\
(n= \\
115)\end{array}$ & $\begin{array}{l}\text { Grade } \\
1 \\
(n= \\
124)\end{array}$ & $\begin{array}{l}\text { Grade } \\
2 \\
(n= \\
50)\end{array}$ & $\begin{array}{l}\text { Grade } \\
3 \\
(n= \\
31)\end{array}$ & $\begin{array}{l}P \\
\text { value }\end{array}$ \\
\hline Age, mean (SD) & $\begin{array}{l}58 \\
(13)\end{array}$ & $\begin{array}{l}57 \\
(13)\end{array}$ & $\begin{array}{l}61 \\
(12)\end{array}$ & $\begin{array}{l}60 \\
(16)\end{array}$ & 0.846 \\
\hline Gender, $\mathrm{n}(\%)$ male & $\begin{array}{l}32 \\
(28 \%)\end{array}$ & $\begin{array}{l}32 \\
(26 \%)\end{array}$ & $\begin{array}{l}19 \\
(38 \%)\end{array}$ & $\begin{array}{l}14 \\
(45 \%)\end{array}$ & 0.104 \\
\hline $\begin{array}{l}\text { Systemic autoimmune disease (except Sjögren } \\
\text { syndrome), } \mathrm{n}(\%)\end{array}$ & $\begin{array}{l}14 \\
(12 \%)\end{array}$ & $\begin{array}{l}14 \\
(11 \%)\end{array}$ & $\begin{array}{l}5 \\
(10 \%)\end{array}$ & $1(3 \%)$ & 0.541 \\
\hline Sjögren syndrome, $\mathrm{n}(\%)$ & $9(8 \%)$ & $\begin{array}{l}14 \\
(11 \%)\end{array}$ & $\begin{array}{l}10 \\
(20 \%)\end{array}$ & $\begin{array}{l}2 \\
(6.5 \%)\end{array}$ & 0.111 \\
\hline Diabetes, n (\%) & $5(4 \%)$ & $9(7 \%)$ & $\begin{array}{l}7 \\
(14 \%)\end{array}$ & $\begin{array}{l}3 \\
(10 \%)\end{array}$ & 0.178 \\
\hline Hypertension, n (\%) & $\begin{array}{l}25 \\
(22 \%)\end{array}$ & $\begin{array}{l}25 \\
(20 \%)\end{array}$ & $\begin{array}{l}17 \\
(34 \%)\end{array}$ & $\begin{array}{l}11 \\
(36 \%)\end{array}$ & 0.102 \\
\hline Thyroid disease, n (\%) & $\begin{array}{l}15 \\
(13 \%)\end{array}$ & $\begin{array}{l}17 \\
(14 \%)\end{array}$ & $\begin{array}{l}6 \\
(12 \%)\end{array}$ & $\begin{array}{l}4 \\
(13 \%)\end{array}$ & 0.991 \\
\hline Cardiac disease, n (\%) & $\begin{array}{l}12 \\
(10 \%)\end{array}$ & $\begin{array}{l}11 \\
(9 \%)\end{array}$ & $3(6 \%)$ & $\begin{array}{l}4 \\
(13 \%)\end{array}$ & 0.726 \\
\hline Sleep disorder, n (\%) & $\begin{array}{l}16 \\
(14 \%)\end{array}$ & $8(7 \%)$ & $\begin{array}{l}7 \\
(14 \%)\end{array}$ & $1(3 \%)$ & 0.104 \\
\hline Parkinson disease, $\mathrm{n}(\%)$ & $8(7 \%)$ & $\begin{array}{l}11 \\
(9 \%)\end{array}$ & $\begin{array}{l}6 \\
(12 \%)\end{array}$ & $\begin{array}{l}4 \\
(13 \%)\end{array}$ & 0.636 \\
\hline Other neurologic disease, $\mathrm{n}(\%)$ & $\begin{array}{l}14 \\
(12 \%)\end{array}$ & $\begin{array}{l}16 \\
(13 \%)\end{array}$ & $\begin{array}{l}5 \\
(10 \%)\end{array}$ & $\begin{array}{l}6 \\
(20 \%)\end{array}$ & 0.663 \\
\hline Pulmonary disease, $\mathrm{n}(\%)$ & $6(5 \%)$ & $4(3 \%)$ & $4(8 \%)$ & $2(7 \%)$ & 0.590 \\
\hline
\end{tabular}

MMP-9, matrix metalloproteinase-9.

Table 4. Comparison of clinical signs and symptoms in semiquantitative results of MMP-9 point-of-care test. 


\begin{tabular}{|c|c|c|c|c|c|}
\hline Parameters & $\begin{array}{l}\text { Grade } 0 \\
(n=115)\end{array}$ & $\begin{array}{l}\text { Grade } 1 \\
(n=124)\end{array}$ & $\begin{array}{l}\text { Grade } 2 \\
(n=50)\end{array}$ & $\begin{array}{l}\text { Grade } 3 \\
(n=31)\end{array}$ & $\begin{array}{l}P \\
\text { value }\end{array}$ \\
\hline OSDI score, mean (SD) & $\begin{array}{l}33.8 \\
(24.0)\end{array}$ & $\begin{array}{l}36.0 \\
(24.1)\end{array}$ & $\begin{array}{l}34.7 \\
(24.7)\end{array}$ & $\begin{array}{l}33.0 \\
(20.9)\end{array}$ & 0.888 \\
\hline VAS scale, mean (SD) & $2.5(2.8)$ & $3.2(2.6)$ & $3.0(3.0)$ & $2.6(2.2)$ & 0.288 \\
\hline Schirmer score, mm, mean (SD) & $4.7(2.4)$ & $4.8(2.5)$ & $4.5(2.5)$ & $4.7(2.4)$ & 0.846 \\
\hline tBUT, sec, mean (SD) & $4.8(3.6)$ & $3.7(2.3)$ & $4.2(3.2)$ & $4.0(2.9)$ & 0.060 \\
\hline tBUT, $\leq 3, \mathrm{n}(\%)$ & $56(48.7)$ & $78(63.0)$ & $28(56.0)$ & $18(58.1)$ & 0.252 \\
\hline Corneal staining, score, mean (SD) & $0.9(1.0)$ & $1.2(1.1)$ & $1.4(1.1)$ & $1.1(1.0)$ & 0.102 \\
\hline Corneal staining, $\geq 2$, n (\%) & $29(25.2)$ & $49(39.5)$ & $23(46.0)$ & $11(35.5)$ & $0.036^{*}$ \\
\hline Conjunctival staining, score, mean (SD) & $1.2(1.8)$ & $1.8(2.1)$ & $1.5(1.8)$ & $1.2(1.5)$ & 0.288 \\
\hline Conjunctival staining, $\geq 2, \mathrm{n}(\%)$ & $37(32.2)$ & $63(50.8)$ & $20(40.0)$ & $11(35.5)$ & 0.457 \\
\hline $\begin{array}{l}\text { Meibomian gland turbidity, grade, mean } \\
\text { (SD) }\end{array}$ & $1.2(0.7)$ & $1.2(0.7)$ & $0.9(0.6)$ & $1.2(0.8)$ & 0.074 \\
\hline $\begin{array}{l}\text { Meibomian gland expression, grade, } \\
\text { mean (SD) }\end{array}$ & $1.2(1.0)$ & $1.3(1.1)$ & $1.3(1.1)$ & $1.5(1.2)$ & 0.660 \\
\hline Meibomian gland dysfunction, $\geq 3, n(\%)$ & $53(46.1)$ & $65(52.4)$ & $24(48.0)$ & $18(58.1)$ & 0.388 \\
\hline
\end{tabular}

MMP-9, matrix metalloproteinase-9; OSDI, ocular surface disease index; VAS, visual analogue scale; tBUT, tear break up time

${ }^{*}$ Statistically significant by linear by linear association chi square test.

Table 5. Clinically significant associations with semiquantitative results of MMP-9 point-of-care test 


\begin{tabular}{|lll|}
\hline Parameters & rvalue & $P$ value \\
\hline Age & 0.094 & 0.093 \\
\hline OSDI score & 0.012 & 0.842 \\
\hline VAS score & 0.074 & 0.199 \\
\hline tBUT & -0.125 & $0.025^{*}$ \\
\hline Schirmer score & -0.017 & 0.766 \\
\hline Meibomian gland dysfunction & 0.033 & 0.613 \\
\hline Conjunctival staining score & 0.060 & 0.281 \\
\hline Corneal staining score & 0.122 & $0.029^{*}$ \\
\hline
\end{tabular}

MMP-9, matrix metalloproteinase-9; OSDI, ocular surface disease index; VAS, visual analogue scale; tBUT, tear break up time

*Statistically significant by linear Spearman rank correlation test.

Table 6. Clinically significant associations with quantitative results of MMP-9 point-of-care test

\begin{tabular}{|lll|}
\hline Parameters & rvalue & $P$ value \\
\hline Age & 0.021 & 0.720 \\
\hline OSDI score & 0.037 & 0.539 \\
\hline VAS score & -0.009 & 0.276 \\
\hline tBUT & -0.015 & 0.293 \\
\hline Schirmer score & -0.019 & 0.748 \\
\hline Meibomian gland dysfunction & 0.034 & 0.622 \\
\hline Conjunctival staining score & 0.021 & 0.720 \\
\hline Corneal staining score & 0.113 & 0.052 \\
\hline
\end{tabular}

MMP-9, matrix metalloproteinase-9; OSDI, ocular surface disease index; VAS, visual analogue scale; tBUT, tear break up time.

Figures 


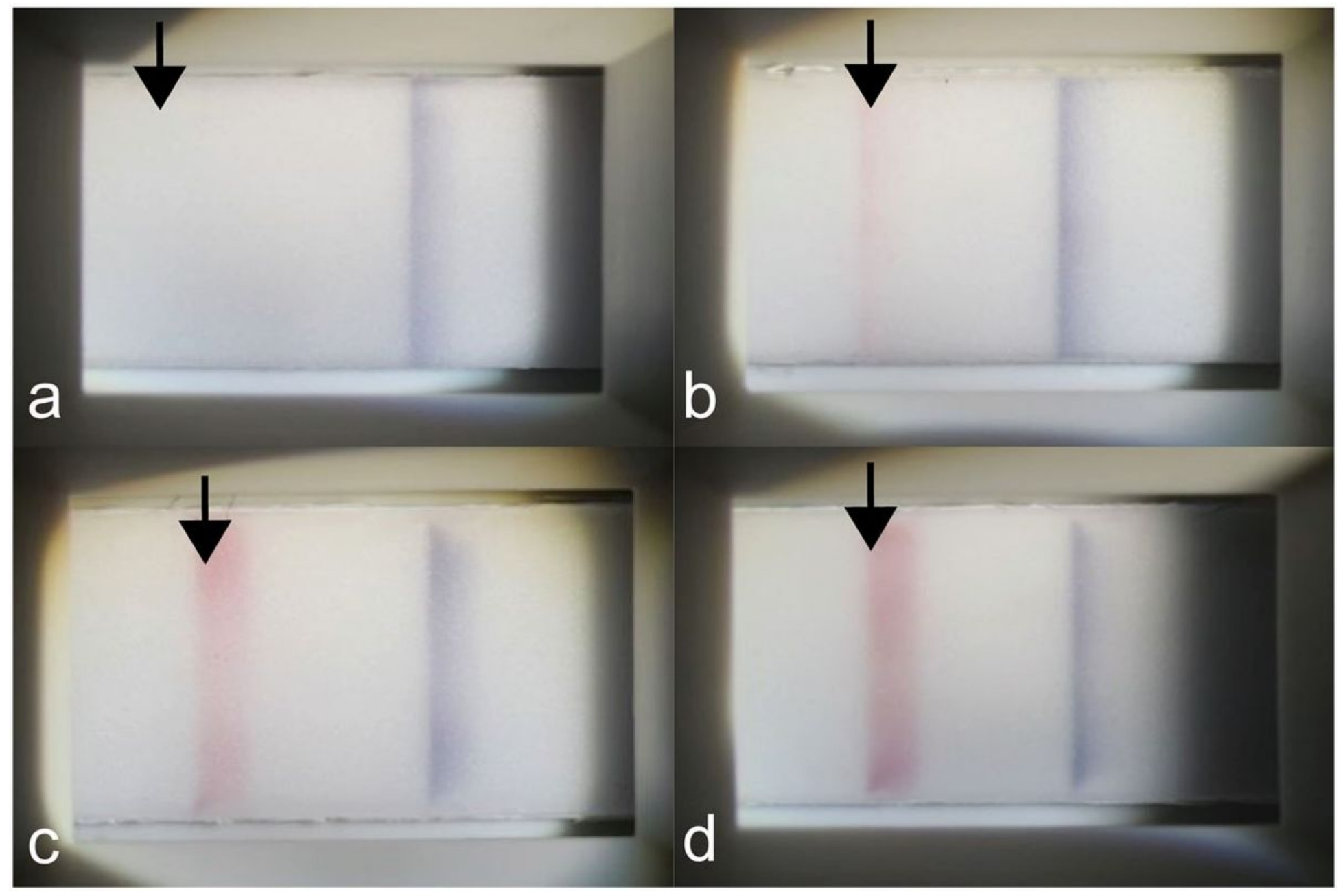

\section{Figure 1}

Semiquantitative analysis of matrix metalloproteinase 9 immunoassay according to the colour intensity of reagent band (arrow). (a) Negative. (b) Weak positive. (c) Moderate positive. (d) Strong positive. 


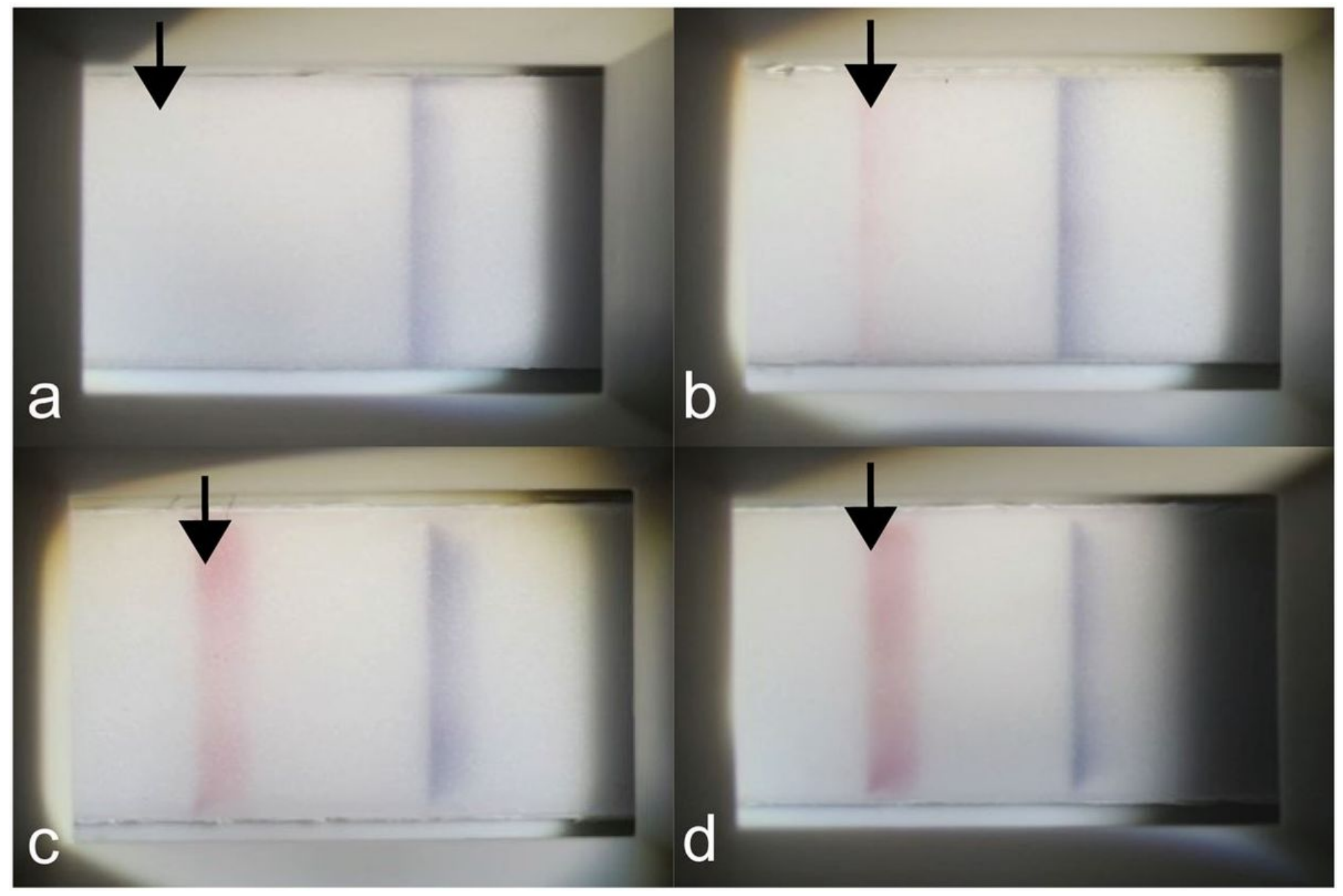

\section{Figure 1}

Semiquantitative analysis of matrix metalloproteinase 9 immunoassay according to the colour intensity of reagent band (arrow). (a) Negative. (b) Weak positive. (c) Moderate positive. (d) Strong positive. 


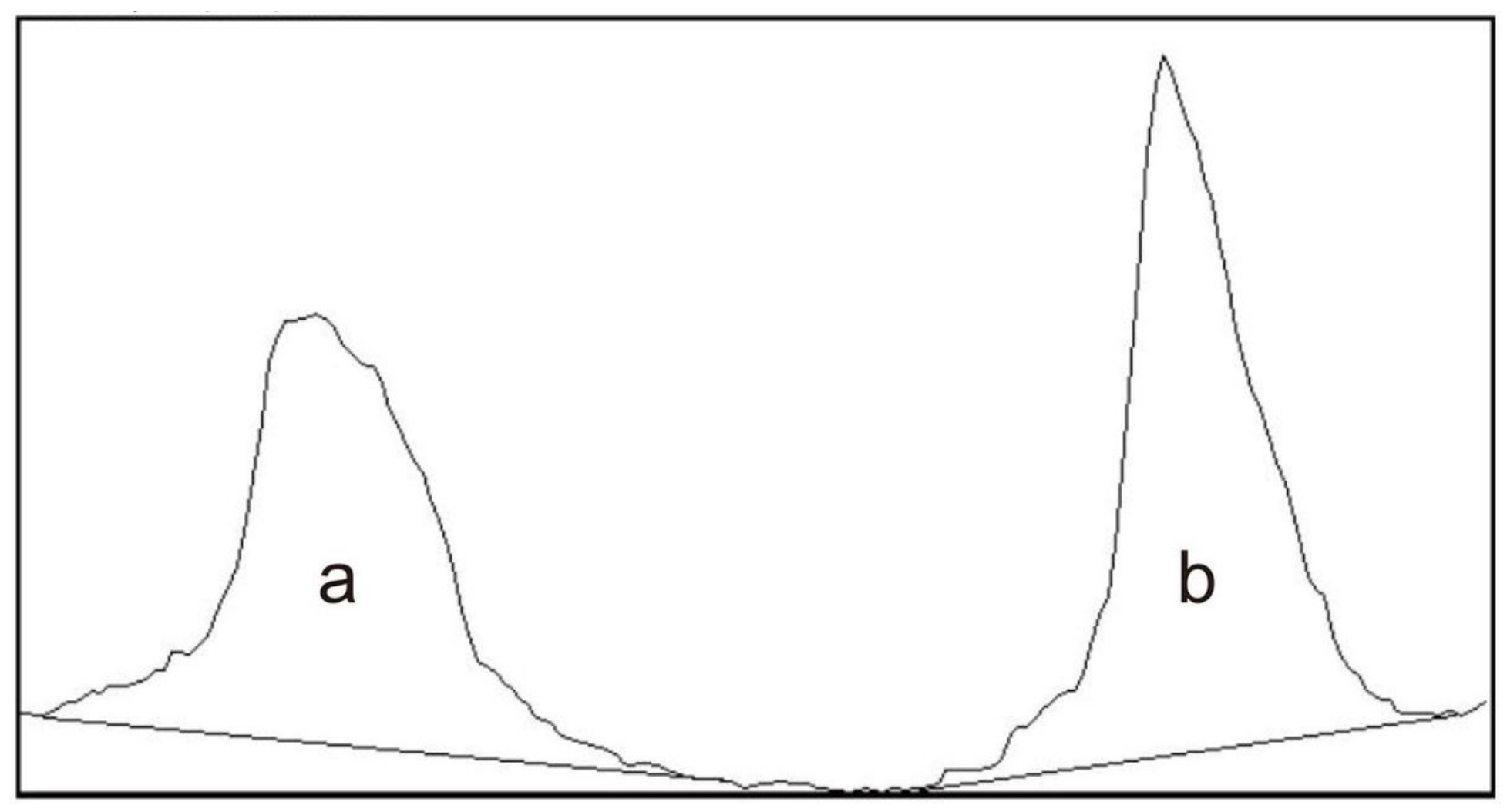

Figure 2

Quantitative analysis of matrix metalloproteinase 9 immunoassay using Image J software version 1.44p (https://imagej.nih.gov/ij/). (a) Reagent band density area. (b) Control band density area.

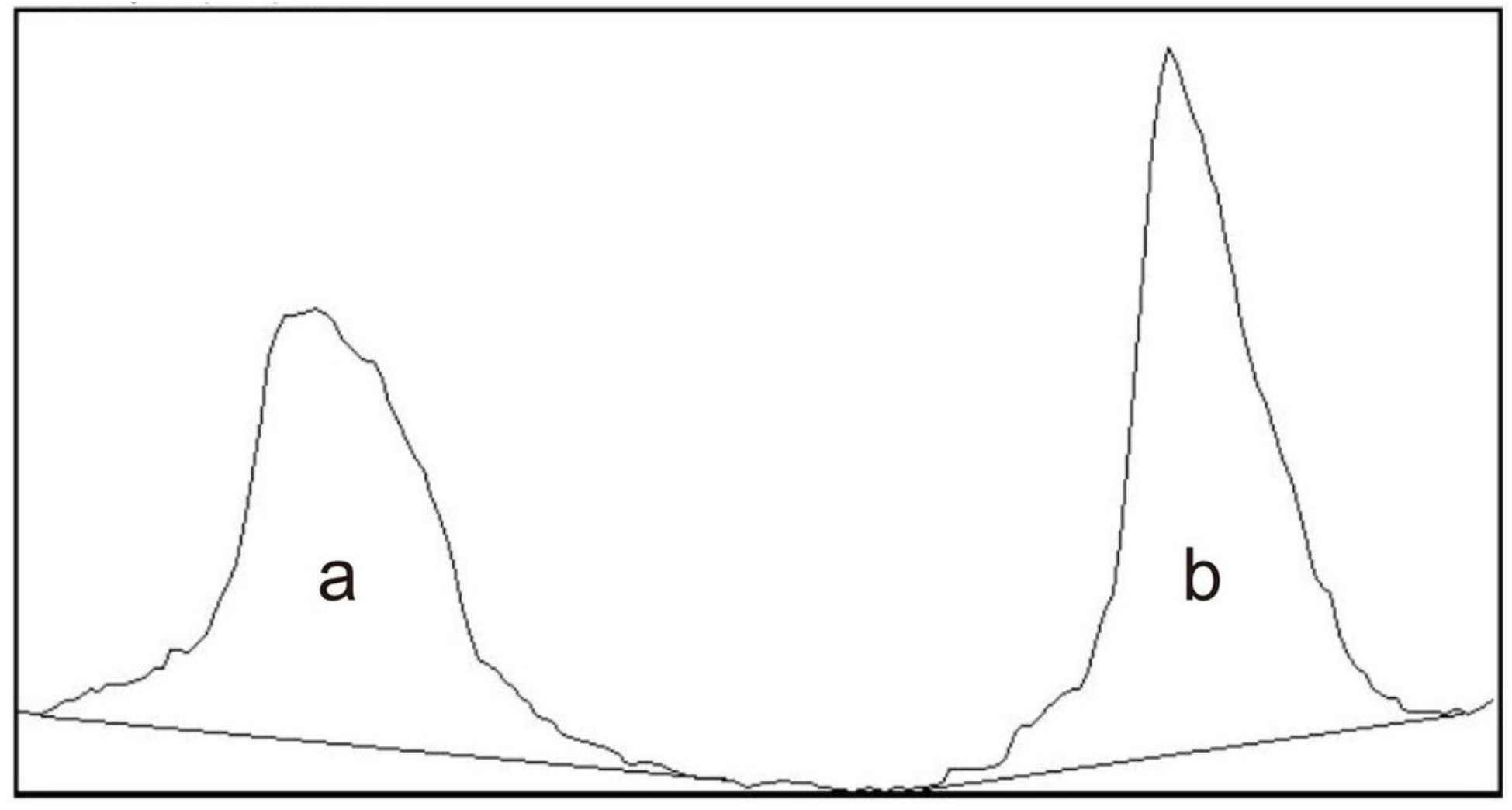

Figure 2 
Quantitative analysis of matrix metalloproteinase 9 immunoassay using Image J software version 1.44p (https://imagej.nih.gov/ij/). (a) Reagent band density area. (b) Control band density area. 